\title{
A Harvest Vehicle with Pneumatic Servo System for gathering a Harvest and its Simulation Study
}

\author{
Katsumi Moriwaki \\ Department of Mechanical Engineering, Daido University, Japan, moriwaki@daido-it.ac.jp
}

\begin{abstract}
A series of works such as harvesting and transporting in a farm is one of such works with so care as not to damage the harvest in order to maintain the value of harvests. We are developing an autonomous cart for gathering a harvest with the bed to be controlled to keep in horizontal level at work and in transit, in order to avoid harvests gathered in particular area of the bed and to keep away from being damaged in harvests. It is proposed a method of autonomous steering control of a harvest vehicle with maintaining the horizontal level of the bed of the cart with air cylinder suspension systems. It is shown that the problem of a level control of vehicle's bed can be formulated as one of optimal control problems. Finally, its simulation study is considered.
\end{abstract}

Keywords: level control of vehicle's bed, pneumatic servo control, autonomous vehicle, terrain farm, harvesting

\section{Introduction}

The work of harvesting of the fields is one of tough works which need the efficiency of materials handling, while it is necessary for guarantee of commodity value to treat crops carefully so that a crack may not be attached to crops. A harvest cart is, therefore, one of necessary apparatuses for both a professional farmer and a urban farmer to lighten their work load to harvest the fields. There are many harvest carts developed and sold in various size, from smalled-sized to mega-sized. In this research, it aims at development of the conveyance cart which can carry a harvest without swaying of the loading bed by performing level surface maintaining control, in order for the crops not to be damaged. We proposed the basic structure for level control of a harvest bed using pneumatic system (Moriwaki, 2012). It was proposed the harvest vehicle with a level-controlled harvest bed and considered the optimal control problem of a level control of a harvest bed (Moriwaki, 2013, 2016). We, furthermore, consider to realize the system of intelligent harvesting which performs autonomous collection of crops in a cultivated land and autonomous carrying them to the crops shed without deterioration of the commodity value of them. Figure 1 shows an examples of harvest vehicle, which carries crops from the cultivated land to the harvest gathering place. The harvest cart often jolts over the rough cultivated land, and crops vibrate on the loading bed of the harvest cart.
Crops collide together or with the wall of a harvest stand, It damages the surface of crops and reduces the commodity value of crops. If crops without cracks can be harvested and shipped, they can be sold for a high price at a market. We have started a development program of a harvest vehicle which can perform harvesting operation and maintain the level surface of its loading bed, and can autonomously go to a harvest collection place.

The suspension model of a vehicle is considered in Section 2. After considering the structure of the loading bed of a harvest vehicle in Section 3, A model of level controlled bed by a pneumatic servo system is proposed in Section 4. The control system of a harvest bed with a pair of pneumatic cylinder is proposed in Section 5, where is also shown some numerical simulations and experimental study. Finally, it is considered the problem of level control of harvest's bed on the terrain farm land in Section 6 .

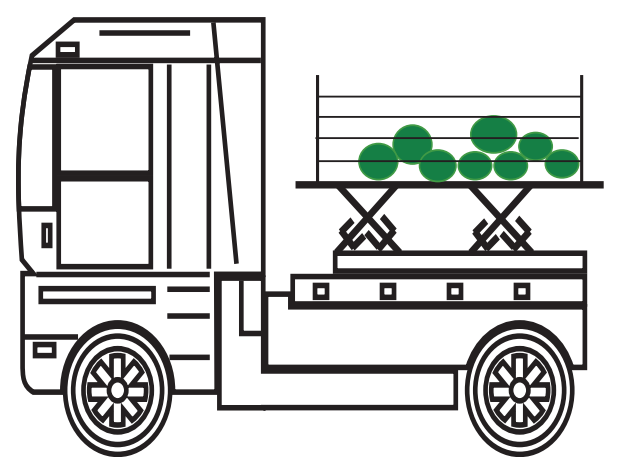

Figure 1. A harvest vehicle with active pneumatic suspension bed.

\section{Suspension model of a vehicle dy- namics}

The control problem of leveling the load bed of a harvest vehicle is deeply relation to the vertical dynamics of a vehicle, its suspension dynamics. The features of car vertical dynamics in $(X-Z)$-plane are described by Figure 2 (Abe, 1992; Andrezejewski and Awrejcewicz, 2005) with active suspension system in which the external control forces is used to suppress the uncomfortable bouncing motion and pitching motion.

The equations of motions for two degrees of freedom in $(X-Z)$-plane with the constant velocity $V$ are 


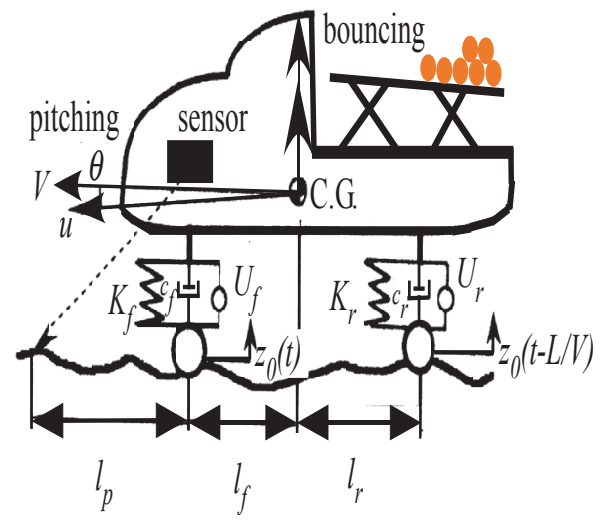

Figure 2. Active suspension control of a harvest vehicle.

$$
\begin{gathered}
\text { bouncing motion : } m \ddot{z}=F_{f}+F_{r} \\
\text { pitching motion : } I_{y} \ddot{\theta}=-l_{f} F_{f}+l_{r} F_{r}
\end{gathered}
$$

where $m$ is the mass of a vehicle, $z$ is the vertical displacement of the center of gravity (CG) of a car(Figure 2), $I_{y}$ is the moment of inertia around y-axis, $\theta$ is the pitching angle of the center of gravity (CG) of a car and the external forces acting on a front wheel and the rear wheel from the road surface are written by $F_{f}$ and $F_{r}$, respectively. The state space model of the vertical dynamics is derived from (1) and (2), where it is assumed that the vehicle is affected from the terrain of the farm land as the unknown input $U_{f}, U_{r}$ and the surface roughness $z_{0 f}=z_{0}(t), z_{0 r}=z_{0}(t-L / V)$, where $L=l_{f}+l_{r}$.

$$
\begin{aligned}
& {\left[\begin{array}{c}
\dot{z} \\
\dot{\theta} \\
\dot{w} \\
\dot{q}
\end{array}\right]=\left[\begin{array}{cccc}
0 & 0 & 1 & 0 \\
0 & 0 & 0 & 1 \\
\tilde{a}_{31} & \tilde{a}_{32} & \tilde{a}_{33} & \tilde{a}_{34} \\
\tilde{a}_{41} & \tilde{a}_{42} & \tilde{a}_{43} & \tilde{a}_{44}
\end{array}\right]\left[\begin{array}{c}
z \\
\theta \\
w \\
q
\end{array}\right]} \\
& +\left[\begin{array}{cc}
0 & 0 \\
0 & 0 \\
\tilde{b}_{31} & \tilde{b}_{32} \\
\tilde{b}_{41} & \tilde{b}_{42}
\end{array}\right]\left[\begin{array}{c}
U_{f} \\
U_{r}
\end{array}\right]+\left[\begin{array}{cc}
0 & 0 \\
0 & 0 \\
\tilde{h}_{31} & \tilde{h}_{32} \\
\tilde{h}_{41} & \tilde{h}_{42}
\end{array}\right]\left[\begin{array}{c}
z_{0 f} \\
z_{0 r}
\end{array}\right]
\end{aligned}
$$

where $w:=\dot{z}, q:=\dot{\theta}$ and

$$
\begin{aligned}
& \tilde{a}_{31}=-\frac{K_{f}+K_{r}}{m}, \quad \tilde{a}_{32}=\frac{K_{f} l_{f}-K_{r} l_{r}}{m} \\
& \tilde{a}_{33}=-\frac{C_{f}+C_{r}}{m}, \quad \tilde{a}_{34}=\frac{C_{f} l_{f}-C_{r} l_{r}}{m} \\
& \tilde{a}_{41}=\frac{K_{f} l_{f}-K_{r} l_{r}}{I_{y}}, \quad \tilde{a}_{42}=-\frac{K_{f} l_{f}^{2}+K_{r} l_{r}^{2}}{I_{y}} \\
& \tilde{a}_{43}=\frac{C_{f} l_{f}-C_{r} l_{r}}{I_{y}}, \quad \tilde{a}_{42}=-\frac{C_{f} l_{f}^{2}+C_{r} l_{r}^{2}}{I_{y}}
\end{aligned}
$$

$$
\begin{aligned}
& \tilde{b}_{31}=\frac{1}{m}, \quad \tilde{b}_{32}=\frac{1}{m} \\
& \tilde{b}_{41}=-\frac{l_{f}}{I_{y}}, \quad \tilde{b}_{42}=\frac{l_{r}}{I_{y}} \\
& \tilde{h}_{31}=\frac{K_{f}}{m}, \quad \tilde{h}_{32}=\frac{K_{r}}{m} \\
& \tilde{h}_{41}=-\frac{K_{f} l_{f}}{I_{y}}, \quad \tilde{b}_{42}=\frac{K_{r} l_{r}}{I_{y}}
\end{aligned}
$$

where

$K_{f}:$ stiffness coefficient for front suspension

$K_{r}$ : stiffness coefficient for rear suspension

$C_{f}$ : damping coefficient for front suspension

$C_{r}$ : damping coefficient for rear suspension

\section{Structure of the loading bed of a harvest vehicle}

It is considered that the structure of the loading platform with the level control mechanism using pneumatic cylinders of an autonomous harvest cart in this section. We propose a control system which maintains a loading platform horizontally while harvested crops is gathered on the loading platform. When crops are taken in and it is stored by the loading platform, there are often for crops to be thrown into the harvest cart and stored randomly on a loading-platform. If the level maintaining of a loadingplatform becomes difficult according to the random loading of crops, a loading-platform inclines and crops may roll in the direction of a lower side, then the damage to the crops caused by collision with other crops or with the wall of the loading-platform may occur, and it may produce deterioration of the commodity value of crops. Moreover, if crops are loaded to the specific side of a loading platform and a loading-platform inclines, the efficiency carrying crops will be affected. In order to prevent such a bad influence to an agricultural harvest work, this research considers a method of the level control of the loading bed of an autonomous harvest cart.

There are many cases in position the load bed with crops (Figure 3). Cases (a) and (b) are situations of the bed on flat field, the bed in (a) is maintained horizontally with the harvest orderly, on the other hand, the bed (b) leans with gathered crops on one side. Case (c) and (d) are sit(3) uations of the bed on terrain field, the bed in (c) leans because of gathered harvest and uneven surface of the field, our aim is to propose the controlled bed to maintain horizontally, whether on uneven field or with gathered crops in ether side. In this paper, it aims at realizing the level control of a loading-platform by using position control of pneumatic cylinders with electromagnetic pressure proportional valves and a computer (Figure 4). In Figure 4, the load $f_{L}$, which is induced for crops to drop on the load platform, presses down the cylinder head, whose mass is denoted by $M$, and the loading platform leans down to either side. The deviation is detected by a height sensor and 
is put into a computer as the reference. The computer calculates the control input $e$, with which a pressure valve controls the cylinder pressure so that the loading platform move back to the horizontally.

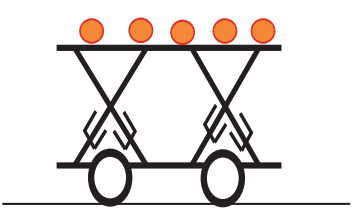

(a) Equal loading of crops

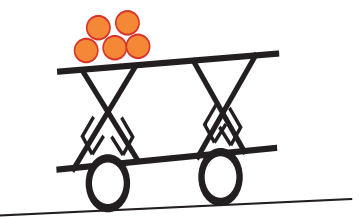

(c) Unequal loading of crops on uneven field.

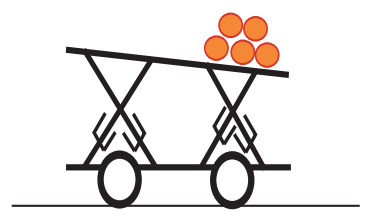

(b) Unequal loading of crops.

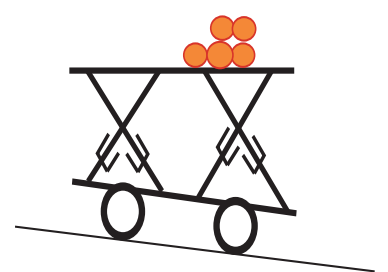

(d) Unequal loading of crops with level control of the bed on uneven field.
Figure 3. Harvest quality managing cart.

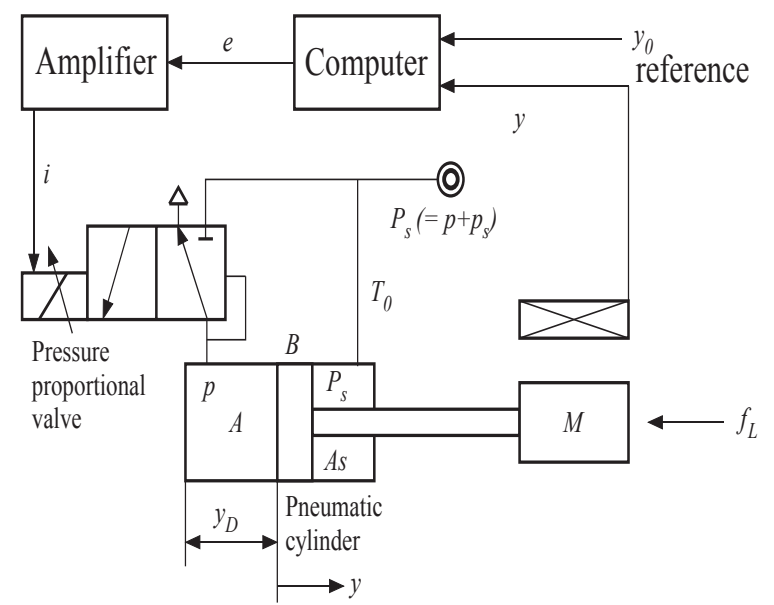

Figure 4. Position control of a pneumatic cylinder with a pressure proportional valve.

\section{Modeling of a pneumatic cylinder with a pressure proportional valve}

A pneumatic cylinder servo control system is expected to be applied to various automation systems, because it has some advantages such as high power/weight ratio, functions of impact absorption and rigorous force control owing to air compressibility. However, the compressibility makes it a high order and nonlinear system, its exact modeling and parameter estimation are not easy (Noritsugu and Takaiwa, 1993; Song, et al., 1997).
There are many paper published about pnematic servo system up to now, almost all of them are, however, dealed with the problem of the position control or force control of a simple pneumatic cylinder. There is few study about the practical control system with the pneumatic servo system (Moriwaki, 2012, 2013, 2016).

\subsection{A model for a pneumatic cylinder}

The air mass flow rate $W[\mathrm{~kg} / \mathrm{s}]$ which flows into a cylinder can be expressed as follows.

$$
W=\frac{1}{R T_{0}}\left\{p \dot{V}+\left(\frac{V}{\kappa}\right) \dot{p}\right\}
$$

where

$$
V=A\left(y_{D}+y\right)
$$

and $A$ is a area of the cylinder $\left[\mathrm{m}^{2}\right], T_{0}$ is an absolute temperature $[K], \kappa$ is the ratio of specific heat of the air $(\approx 1.4)$ and $R$ is the gas constant $\left(=287 \mathrm{~m}^{2} / \mathrm{s}^{2} K\right)$.

The motion of a rod of the pneumatic cylinder is described by the following equation:

$$
M \ddot{y}+B \dot{y}=A p-A_{s} p_{s}+f_{L}
$$

where $y, A_{s}, p, p_{s}, M, B, f_{L}$ are the position of rod in a cylinder, a area of rod-side, a nominal portion of the supply pressure $P_{S}$, a fluctuation portion of the supply pressure $P_{S}$, a inertial mass of the cylinder, a viscous coefficient of friction of the cylinder and a external force, respectively. It is usually assumed that a fluctuation portion of the supply pressure is sufficiently small, then we can put $p_{s}=0$ in (9). Therefore, we obtain the following equation from (9).

$$
M \ddot{y}+B \dot{y}=A p+f_{L}
$$

\subsection{A model for a pressure proportional valve}

It is well known that the transient characteristics of a pneumatic cylinder is remarkably affected to a control flow, inertial mass, death volume of a valve (Tanaka, 1987; Kagawa and Cai, 2010). The servomechanism which controls the cylinder of a single rod type by the three direction type of a pressure proportional valve is used in the proposed system.

The mass flow rate $W_{v}$ may be linearized with respect to the displacement $x_{v}$ of a valve, then we obtain the following equation (Tanaka, 1987; Kagawa and Cai, 2010):

$$
W_{v}=\left(\frac{\partial W_{v}}{\partial x_{v}}\right) x_{v}+\left(\frac{\partial W_{v}}{\partial p}\right) p=k_{v} x_{v}-k_{p} p
$$

where $k_{v}, k_{p}$, are a flow gain, a pressure flow coefficient $\left(k_{p}>0\right)$, respectively. Accompanying the motion of a cylinder to the mass flow rate $W_{v}$ given by (11), the energy equilibrium equation is given by

$$
W_{v}+\frac{1}{R T_{0}} A_{v} p \dot{x_{v}}=W
$$


Equation (12) is transformed and the following (13) is obtained.

$$
W_{v}=\frac{1}{R T_{0}}\left(p A \dot{y}+\frac{V}{\kappa} \dot{p}-A_{v} p \dot{x_{v}}\right)
$$

where $A_{v}$ is the area of a feedback pressure of a valve.

For an electromagnetic force $F_{c}$ of a solenoid, the equation of motion of a pressure valve can be expressed as follows (Tanaka, 1987).

$$
m \ddot{x_{v}}+b \dot{x_{v}}=F_{c}-A_{v} p
$$

where $m$ is the inertial mass of a moving rod of a cylinder and $b$ is the coefficient of viscous damping.

\section{The position control system of a pneumatic cylinder using a pressure proportional valve}

\subsection{A block diagram of a pneumatic servo sys- tem}

The solenoid's electromagnetic force $F_{c}$ can be approximated by the following equation, denoting feedback gains of a cylinder position $y$ and its moving velocity $\dot{y}$ by $g_{f s}, g_{f v}$, respectively,

$$
F_{c} \cong \frac{a_{1}\left(a_{0} y_{0}-g_{f s} y-g_{f v} \dot{y}\right)}{\tau_{0} s+1}
$$

where $a_{1}, a_{0}, \tau_{0}$ denote the electromagnetic force conversion coefficient, the voltage conversion coefficient w.r.t. reference input $y_{0}$, and the time constant of the solenoid.

Combining (10), (11) and (13)-(15), then the position control system is obtained by Figure 5, in which a cylinder position $y$ can be maintained at the reference position $y_{0}$ under an unexpected load (or force) being added to the cylinder. In Figure 5, time constants $\tau_{i}(i=1, \cdots, 4)$ denote $\tau_{1}=\frac{k_{c}}{k_{p}}, \tau_{2}=\frac{m}{b}, \tau_{3}=\frac{A_{v}}{k_{x}}, \tau_{4}=\frac{M}{B}$, respectively, and $k_{x}$ is the flow gain of the valve, $k_{p}$ is the pressure flow coefficient, and $k_{c}$ is the compliance of air pressure. In the usual air pressure servomechanism, $\tau_{0}, \tau_{2}$, and $\tau_{3}$ may be very small and can be ignored (Tanaka, 1987). The time constant $\tau_{4}$ may be changed to $\left(\tau_{4}+\Delta\right)$ depending on the loaded mass.

\subsection{A reduced block diagram of a pneumatic servo system}

If the pneumatic servo system has the condition $\tau_{4}>\tau_{1}$, which means its inertia characteristics are dominant than its volume characteristics in the system, the block diagram shown in Figure 5 can be reduced as Figure 6. In Figure 6, the parameters $\omega_{M}, \zeta_{M}, k_{L p}$ denote the following values respectively.

$$
\omega_{M}=\sqrt{\frac{\left\{A_{v}+\left(a_{1} g_{f v} A\right) / B\right\} k_{x}}{b k_{p} \tau_{4}}}
$$

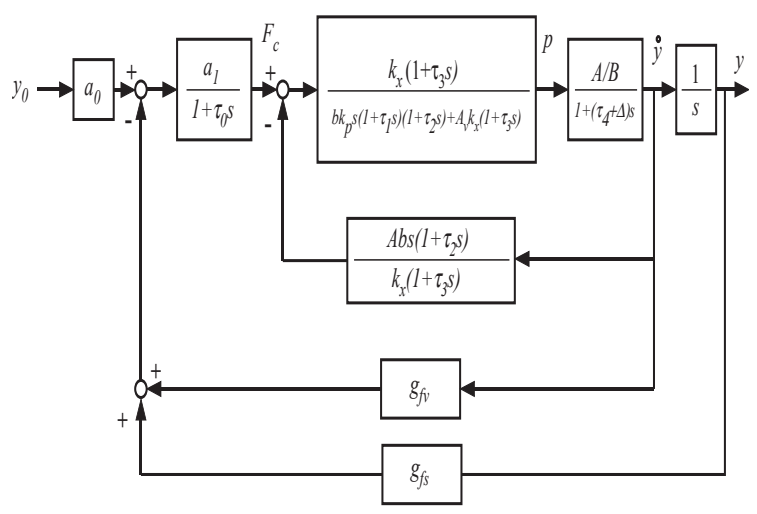

Figure 5. The position control system by a pneumatic cylinder.

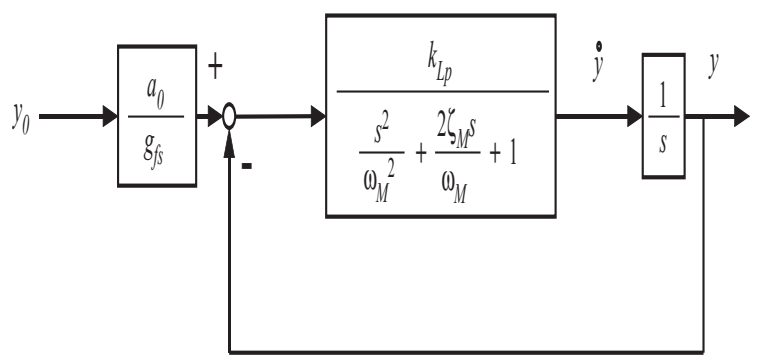

Figure 6. The reduced position control system by a pneumatic cylinder.

$$
\begin{gathered}
\zeta_{M}=\frac{b\left(k_{p}+A^{2} / B\right)+A_{v} k_{x} \tau_{4}}{2 \sqrt{b k_{v} \tau_{4} k_{x}\left\{A_{v}+\left(a_{1} g_{f v} A\right) / B\right\}}} \\
k_{L p}=\frac{\left(a_{1} g_{f p} A\right) / B}{A_{v}+\left(a_{1} g_{f v} A\right) / B}
\end{gathered}
$$

The characteristics of step responce of the pneumatic servo system is analyzed theoretically and the parameter $k_{L p} / \omega_{M}$ is necessary to be set 0.4 or less in order to obtain the undershoot step response (Tanaka, 1987).

\subsection{A state space model for leveling control of the loading bed of a harvest cart}

The loading bed of a harvest cart, which we proposed in this paper, is controlled by a pair of pneumatic cylinders as shown in Figure 7, which is appeared in later section. The structure of dynamics of the proposed level control system is shown in Figure 8.

The rods of pneumatic cylinder (a) and (b) are connected to the loading bed with rotation and bottoms of cylinders are connected to the base of the harvest cart with the angle $\theta$ of inclination. The angle $\theta$ is assumed to be fixed, i.e. $\sin \theta$ is to be constant. The height $z_{a}, z_{b}$ of the both ends of the loading bed are controlled by the position of the rod $y_{a}, y_{b}$, respectively, as

$$
z_{a}=y_{a} \sin \theta, z_{b}=y_{b} \sin \theta
$$




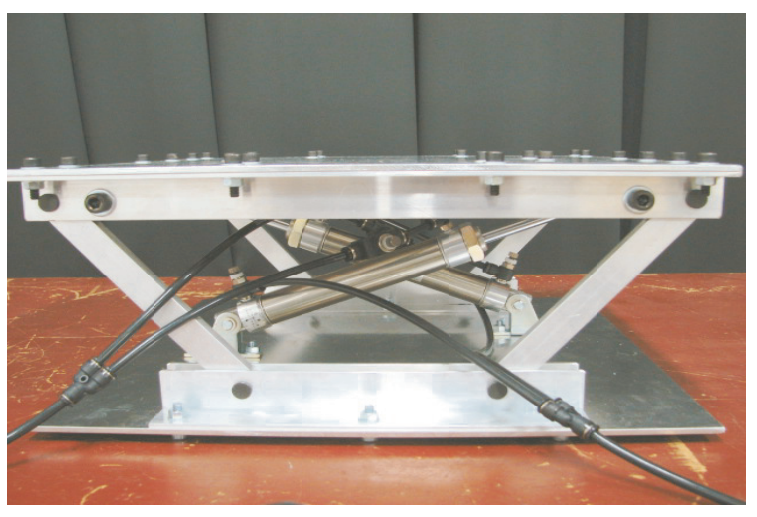

Figure 7. The experimental device for level control of the loading platform.

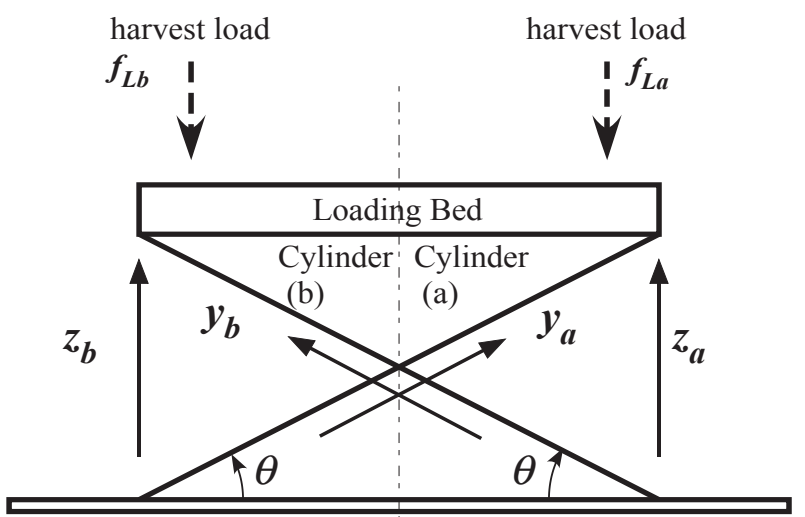

Base of Harvest Cart

Figure 8. The structure of a dynamic model for level control of the loading bed.

with the state space model as follows: $(i=a, b)$

$$
\frac{d}{d t}\left[\begin{array}{l}
y_{i} \\
\dot{y}_{i}
\end{array}\right]=\left[\begin{array}{cc}
0 & 1 \\
0 & -\frac{2 B}{M}
\end{array}\right]\left[\begin{array}{l}
y_{i} \\
\dot{y}_{i}
\end{array}\right]+\left[\begin{array}{c}
0 \\
\frac{2 A}{M}
\end{array}\right] p_{i}+\left[\begin{array}{c}
0 \\
\frac{2 A}{M}
\end{array}\right] f_{L i},
$$

where $p_{i}$ is the control input and $f_{L i}$ is the harvest load. The objective of level control of the loading bed is, therefore, maintain the difference $z_{a}-z_{b}$ to be minimized. That is the difference $v$ defined by

$$
v=\sqrt{\left(z_{a}-z_{b}\right)^{2}}=\sqrt{\left(y_{a}-y_{b}\right)^{2}} \sin \theta
$$

to be reduced as soon as possible.

\subsection{Simulation results of the level control sys- tem}

The harvest is loaded on the loading bed (Figure 8) of the harvest cart unevenly from the farm. We have simulated the level control of the loading bed under (a) the periodical loading from the both ends with different weights

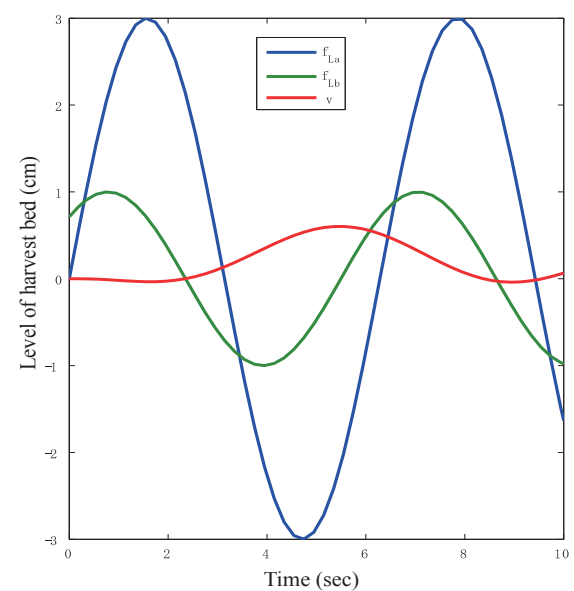

Figure 9. A state of level of the harvest bed under periodical loading.

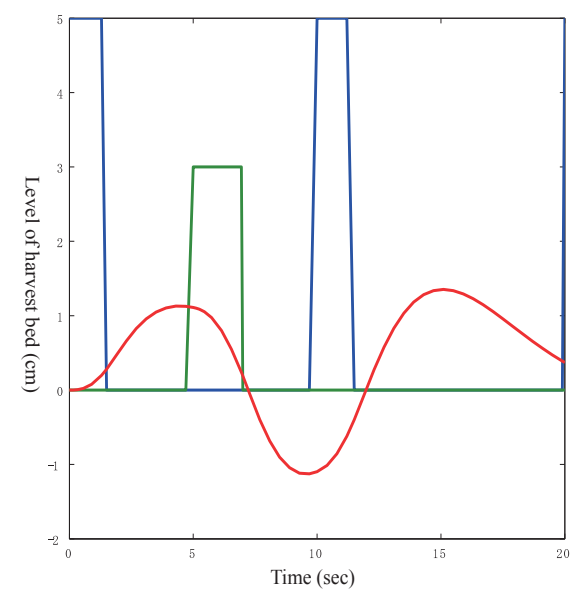

Figure 10. A state of level of the harvest bed under random impulsive loading

and a phase difference and (b) the random impulsive loading from the both ends with different weights and a phase difference. Figure 9 shows the external harvest loadings $f_{L a}, f_{L b}$ and the difference $v$ of the loading bed (21) for the case (a). Figure 10 shows the external harvest loadings $f_{L a}, f_{L b}$ and the difference $v$ of the loading bed (21) for the case (b). In Figures 9, 10 amplitudes of $f_{L a}, f_{L b}, v$ are normalized and scaled.

\section{Leveling control of the harvest bed on the terrain farm land}

The harvest vehicle usually moves on rough terrain to carry crops to the cargo-pickup point at the farm. In this section, we consider the control problem to maintain the level of a harvest bed under the existence of pitching motion of the suspension. Let's assume there is a pitching 


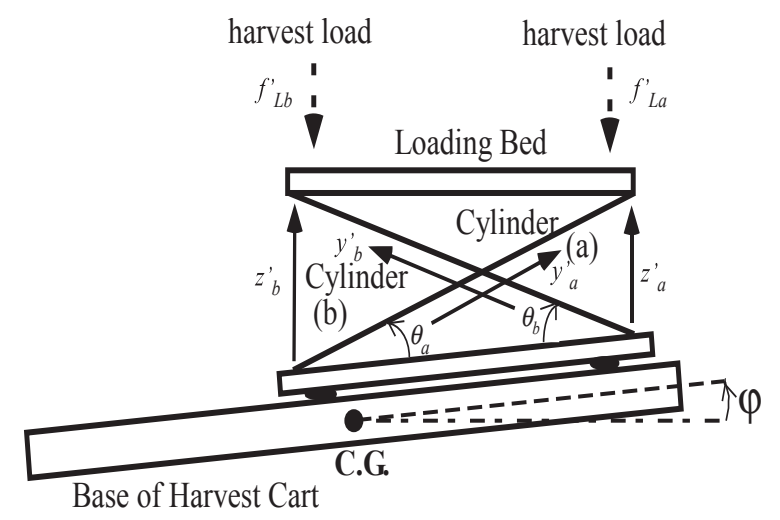

Figure 11. The model of a harvest bed on the platform of the vehicle on rough terrain.

angle $\varphi$, while the harvest vehicle moves. Then the model of a harvest bed under pitching motion is shown in Figure 11.

The height $z_{a}^{\prime}, z_{b}^{\prime}$ of the both ends of the loading bed are controlled by the position of the rod $y_{a}, y_{b}$, respectively, as

$$
z_{a}^{\prime}=y_{a}^{\prime} \sin \theta_{a}, \quad z_{b}^{\prime}=y_{b}^{\prime} \sin \theta_{b}
$$

with the state space model as follows: $(i=a, b)$

$$
\frac{d}{d t}\left[\begin{array}{l}
y_{i_{i}}^{\prime} \\
\dot{y}_{i}^{\prime}
\end{array}\right]=\left[\begin{array}{cc}
0 & 1 \\
0 & -\frac{2 B}{M}
\end{array}\right]\left[\begin{array}{l}
y_{i}^{\prime} \\
\dot{y}_{i}^{\prime}
\end{array}\right]+\left[\begin{array}{c}
0 \\
\frac{2 A}{M}
\end{array}\right] p_{i}+\left[\begin{array}{c}
0 \\
\frac{2 A}{M}
\end{array}\right] f_{L i}^{\prime},
$$

where $p_{i}$ is the control input and $f_{L i}^{\prime}$ is the harvest load. There is the incline of the base of a harvest vehicle, caused by the pitching angle $\varphi$. Therefore, we have to compensate for the incline in order to control the level of the harvest bed. The difference between $z_{a}^{\prime}$ and $z_{b}^{\prime}$ is

$$
\Lambda=l_{r} \sin \varphi
$$

where $l_{r}$ is the distance between the center of gravity (C.G.) and the rear axle of the vehicle (Figure 2).

The objective of level control of the loading bed for this case is, therefore, maintain the difference $z_{b}^{\prime}-z_{a}^{\prime}$ to be equalized to the incline height $\Lambda$, as soon as possible. That is the difference $|\Lambda|-\left|v^{\prime}\right|$ defined by

$$
v^{\prime}=\sqrt{\left(z_{a}^{\prime}-z_{b}^{\prime}\right)^{2}}=\sqrt{\left(y_{a}^{\prime} \sin \theta_{a}-y_{b}^{\prime} \sin \theta_{b}\right)^{2}}
$$

to be reduced as soon as possible. It is not clear in this general case for the solution to level control of the harvest bed of the cart.

\section{Conclusions}

The autonomous harvest cart with level control of loading platform has been proposed in this paper. Crops can be stored in an uneven cultivated land so that reduction in commodity value may not be produced by using the proposed harvest cart. The pneumatic cylinder servo control system is used to control the level of loading platform. The proposed harvest cart is designed so that it can maintain the level of loading platform while loading of crops and following a bumpy road from a farmland.

\section{References}

M. Abe. Vehicle Dynamics and Control. Saikai-do Press, Tokyo, 1992.

R. Andrezejewski and J. Awrejcewicz. Nonlinear Dynamics of a Wheeled Vehicle. Springer, New York, 2005.

Toshiharu Kagawa and Maolin Cai. Measurement and control of compressive fluid -An introduction to air pressure analysis-. Japanese Industrial Publishing Company, Tokyo, 2010.

Katsumi Moriwaki. An autonomous cart for gathering a harvest and its control. In Proc. of 2012 ISCIE Conference, pages 35-36. ISCIE, Osaka, 2012.

Katsumi Moriwaki. Level control for the Loading Bed of a Harvest Cart by the Pneumatic Servo System and its Simulation Study. In Proc. of 2013 8th EUROSIM, pages 90-94. SIM, Cardiff, UK, 2013. doi:10.1109/EUROSIM.2013.26.

Katsumi Moriwaki. A harvest vehicle with pneumatic servo system for gathering a harvest and its control. ScienceDirect, 49-21:460-466. ELSEVIER, 2016.

M. Nagai, A. Okada, K. Komoridani, Y. Suda, K. Tani, H. Amijima, S. Nakajiro, H. Harada, M. Miyamoto, and H. Yoshioka. Dynamics and Control of Vehicles. Yoken-do Press, Tokyo, 1999.

Toshiro Noritsugu and Masahiro Takaiwa. Robust control of a pneumatic servo system using disturbance observer. Trans. of SICE, 29:86-93. SICE, Tokyo, 1993.

Toshiro Noritsugu and Masahiro Takaiwa. Positioning control of pneumatic servo system with pressure control loop using disturbance observer. Trans. of SICE, 31 :1970-1977. SICE, Tokyo, 1995.

Junbo Song, Kazunori Kadowaki and Yoshihisa Ishida. Practical model reference robust control for pneumatic servo system. Trans. of SICE, 33:995-1001. SICE, Tokyo, 1997.

Hirohisa Tanaka. Digital control of a hydraulic system and a pneumatic system and its applications. Kindai Tosho Press, Tokyo, 1987. 